\title{
Realidad de la investigación en salud en Chile: participación de los médicos en el Fondo Nacional de Investigación (FONIS) en la última década.
}

\author{
Tomás P. Labbé ${ }^{1,5,6}$, M. Francisca Barake ${ }^{2,5,6}$, M. Florencia Álamos ${ }^{1,5,6}$, Cleofe G. Molina 3,4,6, Juvenal A. Ríos $2,4,5,6^{*}$
}

Resumen: Chile es uno de los países de la OCDE que menos invierte en ciencia y tecnología, y la investigación en salud no está exenta de este problema. Existe evidencia reportada en publicaciones nacionales de que la participación de médicos en la investigación ha experimentado un marcado retroceso en las últimas décadas. El objetivo de este artículo es analizar específicamente la participación de los médicos como investigadores responsables en proyectos financiados por el Fondo Nacional de Investigación en Salud (FONIS). Este programa fue diseñado para promover investigaciones que resuelvan problemas prioritarios de salud y de esa manera entregar enfoques diagnósticos y terapéuticos a la medida del fenotipo local. Nuestros análisis muestran un estancamiento real y una disminución porcentual, misma tendencia que estudios previos. La implementación de recomendaciones internacionales en el campo de la medicina trasnacional en las políticas de salud nacionales podría revertir el aparente proceso de retirada de los médicos de las actividades de I+D, lo que beneficiaría la salud de los pacientes y reduciría los costos para el sistema, de, por ejemplo, la importación de la terapéutica.

Palabras clave: Investigación; salud; inversión; diagnóstico; medicina trasnacional; desarrollo.

Abstract: Chile is one of the OECD countries that invests less in science and technology, and health research is not exempt from this problem. Evidence reported in national publications shows that the participation of physicians in research has experienced a marked decline in recent decades. The objective of this article is to specifically analyze the participation of physicians as responsible researchers in projects funded by the National Fund for Health Research (FONIS). This program was designed to promote research that addresses priority health issues and thereby delivers diagnostic and therapeutic approaches tailored to the local phenotype. Our analyzes show a real stagnation and a percentage decrease, same trend as previous studies. The implementation of international recommendations in the field of translational medicine in national health policies could reverse the apparent process of withdrawal of physicians from R\&D activities, which would benefit the health of patients and reduce the costs for the system, for instance, in the ones implied of importing therapeutics.

Keywords: Research; health; investment; diagnosis; translational medicine; development.

Fecha de envío: 19 de junio de 2017 - Fecha de aceptación: 31 de agosto de 2017

\section{Consideraciones generales}

Actualmente nuestro país enfrenta una crisis en el área del desarrollo científico y tecnológico. Entre los factores objetivos que se identifican con mayor claridad destacan: una baja inversión en investigación y desarrollo (I+D), que actualmente fluctúa en torno al 0,4\% del Producto Interno Bruto (PIB) y se encuentra muy por debajo del promedio de los países de la Organización para la Cooperación y Desarrollo Económicos, u OECD por sus cifras en inglés (2,3\%) (Brahm, 2016; OECD, 2014). Preocupantemente, esta cifra no ha crecido en los últimos 40 años. Por otro lado, contamos con una limitada diversificación de la matriz productiva, acompañada de una alta dependencia de la exportación de materias primas como el cobre (OECD, 2014); una baja población de investigadores cada mil empleados, en comparación con otros países, lo que, según

(1) Facultad de Medicina. Pontificia Universidad Católica de Chile

(2) Facultad de Ciencias Biológicas. Pontificia Universidad Católica de Chile

(3) Hospital Roberto del Río

(4) Ministerio de Salud de Chile

(5) Capítulo de Médicos Científicos

(6) Observatorio de Pertinencia e Innovación en Salud, Colegio Médico de Chile AG.

"Autor de Correspondencia:jriosleal@gmail.com 
los más recientes datos de la OECD, probablemente sea consecuencia del limitado gasto en investigación (OECD, 2014) (Figura 1). A lo anterior se suma el déficit en los puestos de trabajo para una población de científicos que se está formando dentro y fuera del país (Gonzalez \& Jiménez, 2014; Morales \& Fernandez, 2017). Otro aspecto que impacta en el desarrollo e innovación es que, en Chile, al igual que en el resto de Iberoamérica, las universidades, que son el motor científico del país, producen más ciencia básica que ciencia aplicada (Cruz, 2016).

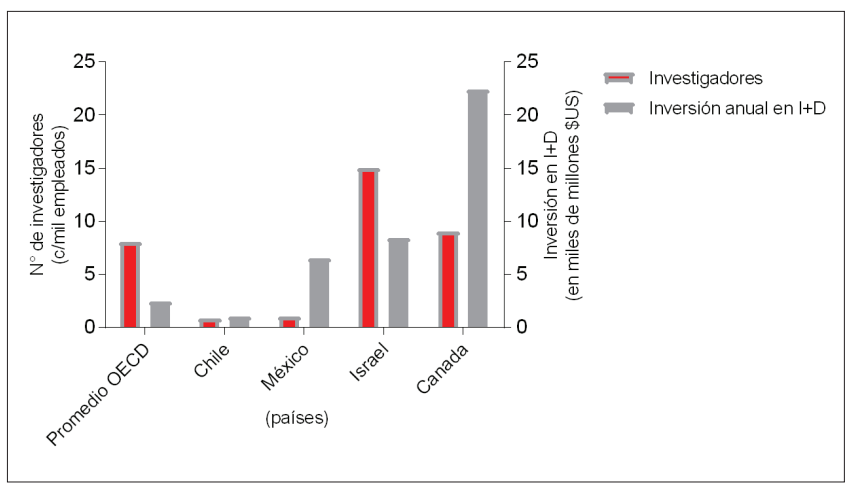

Figura 1: Datos OCDE respecto a $\mathrm{N}^{\circ}$ de investigadores e inversión en I+D. Número de Investigadores e Inversión en I+D. En todos los niveles de comparación, Chile tiene un menor número de investigadores e invierte menor cantidad de dinero en Investigación. Como muestra el gráfico, la comparación es desventajosa ya sea frente a líderes mundiales en $\mathrm{I}+\mathrm{D}$, otros países latinoamericanos y el promedio de la OCDE.

En el escenario anterior, la investigación en salud enfrenta las mismas dificultades que el panorama científico nacional, sin embargo, a esta se le suman factores adicionales que pretendemos ahondar en este trabajo.

La escasa producción e influencia de las investigaciones locales en el área biomédica se ve reflejada en las guías clínicas de las patologías incluidas en las Garantías Explícitas en Salud (GES). Al medir la presencia de citas bibliográficas nacionales en las referencias utilizadas por estos instrumentos, se observó que solo el $2,8 \%$ del total de las referencias corresponden a publicaciones de autores nacionales financiados por fondos estatales como el FONIS; el resto $(98,2 \%)$ corresponde a trabajos internacionales cuyas poblaciones estudiadas probablemente difieren del fenotipo y realidad de nuestros pacientes (Núñez et al., 2014).

La Organización Panamericana de la Salud (OPS), ya en 1991, sugirió al MINSAL que desarrollara una política coordinada de ciencia y salud (Montoya, 2013). En la misma línea, en 1993 se crea en Ginebra el "Forum on Health Research" enfocado a corregir la brecha "10/90". Esta brecha hace referencia a que solo el $10 \%$ de los fondos mundiales para la investigación científica en salud se utilizan para el $90 \%$ de las enfermedades más letales de la humanidad (PAHO, 2008). En este sentido, desde el año 2008, el departamento de estudios del MINSAL, coordinó una instancia para establecer las líneas prioritarias en Chile. De dicha instancia emergen tres grandes líneas de investigación que, a juicio de los expertos, deben financiarse dirigidamente: i) Gestión y evaluación de programas de salud, ii) Patologías de alta prevalencia y iii) Patologías relacionadas con inequidades sociales (Armas et al., 2010).

En esta línea, se debe destacar la importancia del "médico investigador" como un profesional capaz de traducir rápidamente los descubrimientos biológicos en aplicaciones concretas para el tratamiento y prevención de los pacientes, actividad actualmente conocida como "medicina traslacional" (Horton, 1999; Moskowitz \& Thompson, 2001; Saenz et al., 2011). Por otro lado, el "médico investigador" es un agente idóneo para detectar aquellos problemas de salud que necesiten ser resueltos y estudiarlos.

La situación de los médicos investigadores en Chile ha sido analizada ya en el 2005 por Salas y Rigotti en ciencia básica, que hicieron un análisis de la participación de los médicos como IR (Investigadores Responsables) de proyectos financiados por FONDECYT para el período entre 1984 - 2003. El estudio reveló una caída de alrededor del $30 \%$ de la participación de los facultativos entre esos años por Salas \& Rigotti $(2005,2006)$.

Para enfrentar de manera dirigida el desafío de potenciar la innovación y el desarrollo en salud humana, el año 2000, un grupo de expertos del Ministerio de Salud y de CONICYT llegó a la conclusión de que en Chile existía una masa de capital humano avanzado apto para incorporar e integrar conocimiento científico biológico a políticas públicas de salud. Por lo anterior, el 31 de marzo de 2004 se oficializó la creación del Fondo Nacional de Investigación en Salud (FONIS), que hasta el 2014 ha entregado un total de $\$ 6.450$ millones de pesos desde su creación (FONIS, 2014).

El objetivo principal de los proyectos FONIS es generar conocimiento en salud aplicada, tanto para construir políticas sanitarias como para sistematizar decisiones y conductas clínicas en problemas de relevancia para la población. Pueden participar todo tipo de profesionales de la salud tanto médicos como no médicos. Cabe señalar que hasta el 2015 el monto máximo de financiamiento otorgado por este concurso asciende a 30 millones de pesos por un período de 24 meses por investigación, recién en el 2016 podemos constatar una expansión a 60 millones por 24 meses.

En el caso particular de los médicos con formación científica, varios autores han señalado que constituyen el profesional más idóneo 
Labbé et al.

para generar y guiar preguntas científicas que sean clínicamente relevantes (Goldstein \& Brown, 1997; Varki \& Rosenberg, 2002). Como el objetivo principal del FONIS es potenciar la salud aplicada y siendo la medicina traslacional parte de esta, los médicos investigadores deberían tener un grado no menor de participación en estos proyectos de ciencia aplicada. Es por esto que el objetivo de este trabajo es describir cómo ha evolucionado la participación del médico como IR de proyectos financiados por este fondo en particular. Para ello se analizó el perfil de los IR de los proyectos aprobados por FONIS entre el período 2003-2014, a partir de la base de datos disponible de FONIS (CONICYT, n.d.) y buscando la profesión en la superintendencia de salud y otras fuentes. Se agrupó las distintas profesiones en las siguientes dos categorías: médicos (MD) y no-médicos (no-MD), donde entraron profesionales como enfermeras, kinesiólogos, psicólogos, otros profesionales de la salud y también en algunos casos, antropólogos. De los 271 proyectos analizados, fue posible identificar la profesión de todos los IR, (los períodos 2015-2016 aún no estaban disponibles, cuando esta investigación comenzó).

\section{Análisis global de los proyectos adjudicados en los últimos 10 años a través del FONIS}

En los 10 años estudiados se ha financiado a un total de 271 proyectos, con un promedio de 27,1 proyectos por año. Es posible identificar dos fases en la evolución de la cantidad de proyectos FONIS adjudicados. El primer período incluye los años comprendidos entre 2004 y 2009 , donde, más allá del aumento transitorio de la adjudicación en 2005 (25 proyectos), la cantidad total de proyectos tiende a mantenerse estable, con alrededor de 20 proyectos por año. El segundo período, desde 2010 en adelante, muestra un sostenido aumento en la cantidad de proyectos FONIS, de tal manera que al final del período estudiado, la cantidad absoluta de proyectos de investigación con esta fuente de financiamiento alcanza aproximadamente el doble de los valores iniciales (39 proyectos el 2013), con una tasa promedio de aumento anual de $8,6 \%$ (Figura 2).

El total de fondos distribuidos durante este período de 10 años corresponde a $\$ 6.450$ millones de pesos. En el año 2004 se repartió un total de $\$ 340$ millones de pesos y en el 2013 , un total de $\$ 1.010$ millones de pesos, presentando un aumento de un 196\% de los fondos a distribuir. Esta cifra ajustada según los cambios observados en la inflación entre el año 2004- 2014 corresponde a un 118\%.

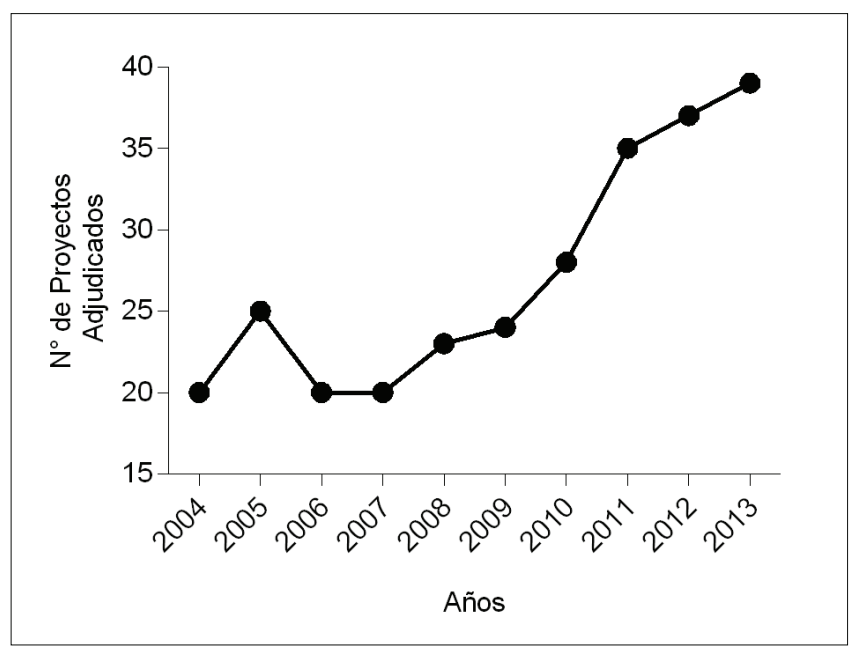

Figura 2: FONIS en el Período 2004-2013. Número total de proyectos FONIS adjudicados por año ha tenido un aumento desde el año 2007.

\section{Contribución del médico como Investigador Responsable (IR) en el período 2004-2013}

Con respecto a la participación de médicos como IR de los proyectos adjudicados en el mismo período, es posible observar un claro estancamiento del número absoluto de proyectos adjudicados, el cual alcanza en promedio 13 proyectos por año (Figura 3), pese al sostenido aumento en la cantidad de proyectos adjudicados ya comentado en la Figura 2.

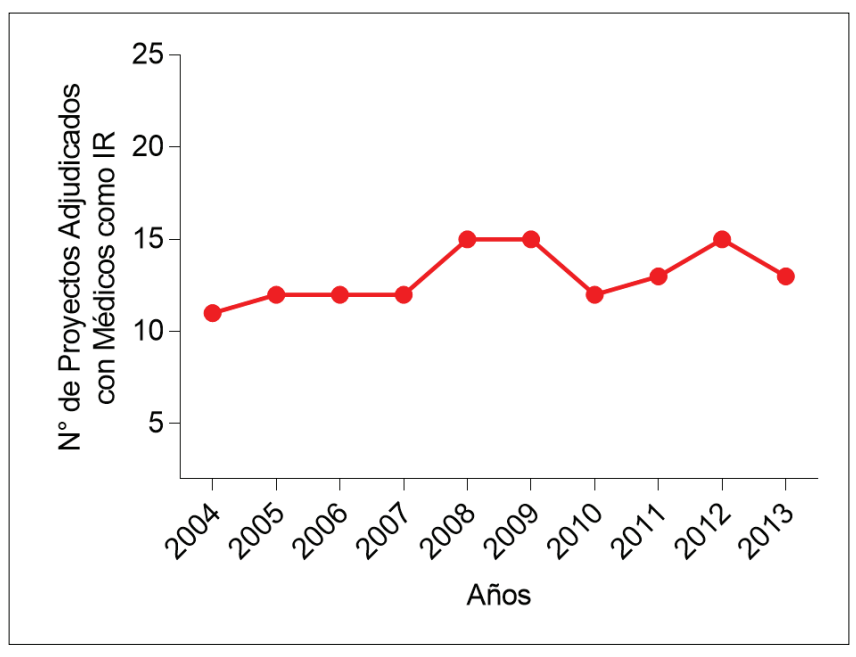

Figura 3: MD como IR de Proyectos FONIS 2004-2013. El número de médicos como IR que se han adjudicado proyectos FONIS se ha mantenido constante a lo largo del tiempo. 
Al comparar la variable anterior con los cambios en la cantidad de proyectos de investigación que son encabezados por profesionales de una formación diferente a la medicina, queda en evidencia un aumento en la representación porcentual de estos en comparación a los proyectos científicos encabezados por médicos, donde el porcentaje desde 2008 en adelante cae drásticamente del 65\% al 33\% para el año 2013. En este último período es interesante destacar una disminución promedio de un 10,6\% anual en la participación de los médicos en este fondo de investigación (Figura 4).

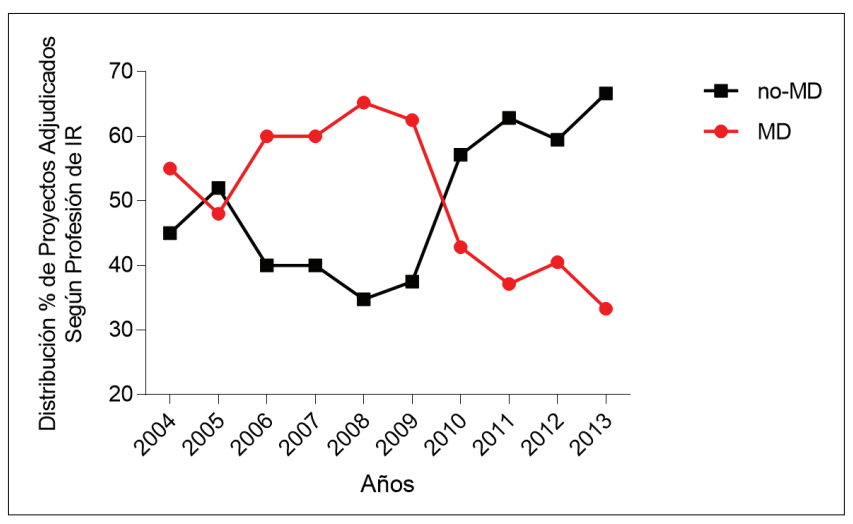

Figura 4: IR de Proyectos FONIS 2004-2013. El porcentaje de proyectos FONIS adjudicados por médicos ha ido en descenso constante desde el año 2008 hasta la fecha.

\section{Conclusiones y recomendaciones}

Uno de los grandes puntos a destacar en este análisis es la disminución porcentual de los médicos como IR de proyectos FONIS. En esta línea, es mandatorio reconocer que, en números absolutos, la presencia de médicos en el desarrollo de proyectos financiados por FONIS tiende a mantenerse estable. No obstante, lo anterior, sería esperable que, al aumentar el número de iniciativas anualmente financiadas, la participación de los médicos como IR se hubiera mantenido también estable en términos porcentuales. Lo anterior plantea que diversas condiciones pueden estar impidiendo que la participación de los profesionales en estudio aumentara.

Por un lado, está la alta carga asistencial a la cual deben enfrentarse los médicos en su quehacer diario, la cual, sumado a la falta de incentivos, muchas veces limita la posibilidad de realizar investigación de calidad de forma paralela. Por otro lado, existe un déficit de "médicos científicos" que cuenten con la formación idónea para realizar investigación clínica basada en sólidos principios básicos de calidad y vanguardia que permitan una rápida transferencia del conocimiento desde el ámbito básico al clínico. En este contexto, Salas y Rigotti discuten las causas que explican el inminente proceso de extinción de los médicos investigadores, las cuales son: i) Bajo incentivo económico, ii) Escasa experiencia investigativa en el pregrado iii) Nula formación científica durante formación del especialista clínico y iv) Escaso interés de médicos en programas de doctorado de acuerdo a Salas \& Rigotti (2005).

Desde otra perspectiva, la reciente experiencia británica, demuestra que la creación de un programa especial de investigación en salud influye positivamente en el sistema de salud. La instancia no está únicamente enfocada a la inyección de más recursos, los cuales han crecido en un 400\%, sino a la implementación de las siguientes 4 medidas: i) Creación de redes entre investigadores de salud, ii) Construcción de una plataforma seria donde publicar estudios que no tienen impacto internacional, iii) Valorización de estudios que analicen la costo-efectividad de las medidas terapéuticas en forma periódica y iv) Financiamiento dirigido a estudios que aborden problemas de salud local. Este programa actualmente ha influido en la toma de decisiones a nivel político, en la práctica clínica y en los pacientes. Por último, y en línea con el objetivo de este artículo, cabe destacar que la experiencia británica ha considerado particularmente la inserción y retención sistemática de investigadores en su sistema público de salud (Guthrie et al., 2015).

Considerando que tras la formación de alianzas básico-clínicas han existido casos de éxito tan emblemáticos como lo es la derrota de la desnutrición infantil en el año 1950, no cabe duda de que este es un camino que como sociedad debiésemos potenciar. En aquella época se enfrentó con éxito la enorme tasa de muerte infantil, entre cuyos múltiples factores destacaba el inadecuado manejo de la diarrea en los lactantes. Derivado de la afortunada convergencia de Herman Niemeyer (bioquímico), Fernando Mönckeberg (nutriólogo) y Fernando Beas (pediatra) se pudieron implementar las primeras fórmulas hidratantes, desde el laboratorio de investigaciones pediátricas y manufacturadas en territorio nacional. Esto, más un conjunto de medidas de salud pública, lograron disminuir la mortalidad infantil a niveles que le han dado al sistema de salud chileno reconocimiento internacional (Szot, 2002; Jiménez, 2010).

Creemos que políticas orientadas en este sentido y que no requieren demasiada inversión de recursos pueden ayudar a fomentar el rol del investigador clínico y al mismo tiempo ser favorables para el sistema de salud afectando positivamente la salud de la población.

\section{Agradecimientos}

Agradecemos al Consejo Regional de Santiago del Colegio Médico de Chile A.G. y a la Mesa Directiva Nacional por destinar fondos a los capítulos para el incentivo de publicaciones científicas con impacto en salud pública.

Este trabajo además se pudo realizar gracias el financiamiento de los autores TPL, MFB, MFA y JAR, quienes fueron beneficiados por la Beca de Doctorado Nacional de CONICYT del Ministerio de Educación del Gobierno de Chile. 
Labbé et al.

Ninguno de los autores (TPL, MFB, MFA, CGM y JAR) presenta conflicto de intereses.

\section{Referencias}

Armas, R., Torres, A., Arriagada, J., Muñoz, F., Salinas, R., \& Crocco, P. (2010). Proceso para priorizar las líneas de investigación esencial de interés para el Ministerio de Salud de Chile. Rev Med Chile, 138, 401-405.

Brahm, J. (2016). Ciencia e investigación en Chile. Una deuda pendiente. Abriendo El Debate. Recuperado de http://www.academia. edu/20944560/Ciencia_e_investigación_en_Chile._Una_deuda_pendiente

CONICYT. (n.d.). Fonis | Fondo Nacional de Investigación y Desarrollo en Salud. Recuperado 13 de Junio 2017, desde http://www.conicyt.cl/fonis/Cruz A. (2016). Innovación de Base Científica-Tecnológica desde las Universidades de Iberoamérica. Journal of Technology Management \& Innovation, 11(4), 1-4.

FONIS. (2014). 10 años apoyando la investigación aplicada en salud en Chile. Santiago. Recuperado de http://www.conicyt.cl/fonis/ files/2014/12/Fonis-10-anios-de-apoyando-la-investigación-aplicada-en-salud-en-Chile.pdf

Goldstein J \& Brown M (1997). The clinical investigator: bewitched, bothered, and bewildered-but still beloved. The Journal of Clinical Investigation, 99(12), 2803-2812.

Gonzalez, H., \& Jiménez, A. (2014). Inserción Laboral de Nuevos Investigadores con Grado de Doctor en Chile. Journal of Technology Management \& Innovation, 9(4), 132-148.

Guthrie, S., Bienkowska-Gibbs, T., Manville, C., Pollitt, A., Kirtley, A., \& Wooding, S. (2015). The impact of the national institute for health research health Technology assessment programme, 20032013: A multimethod evaluation. Health Technology Assessment, 19(67), 1-291.

Horton, B. (1999). From bench to bedside... research makes the translational transition. Nature, 402(6758), 213-215.

Jiménez J. (2010). Construir Políticas Infantiles desde la Ciencia y la Mística. Revista Chilena de Pediatría. 81 (4), 295-299.
Montoya C. (2013). La salud dividida: Chile 1990-2012: ideología, debates y decisiones acerca de la salud: lo que dicen los documentos del período de los gobiernos de la Concertación (1 a edición). Santiago de Chile: Ceibo Ediciones. Recuperado de http://www.bcn.cl/ catalogo/detalle_libro?bib=252833

Morales N \& Fernández I. (2017). Chile unprepared for Ph.D. influx Science, 356(6343), 1131-1132. Recuperado de http://science. sciencemag.org.ezproxy.puc.cl/content/356/6343/1131.2/tab-pdf

Moskowitz, J. \& Thompson, J. (2001). Enhancing the clinical research pipeline: training approaches for a new century. Academic Medicine : Journal of the Association of American Medical Colleges, 76(4), 307-315.

Núñez P, Torres, A \& Armas, R. (2014). ¿Se sustentan las Guías GES en trabajos científicos financiados por los fondos de CONICYT? Rev Med Chile, 142, 1540-1546.

OECD. (2014). OECD Factbook 2014: Economic, environmental and social statistics. OECD Publishing. Recuperado: http://www.oecd-ilibrary.org/economics/oecd-factbook-2014_factbook-2014-en

PAHO. (2008). 1 ra Conferencia Latinoamericana sobre Investigación e Innovación para la Salud: Informe. Rio de Jaeneiro. http://doi.org/ ISBN 978-92-75-33254-2

Saenz, C., Saenz, M., \& Saenz, R. (2011). Medicina traslacional. Del laboratorio a la clínica y de la clínica a la acción. Gatroenterol. Latinoam, 22(3), 263-264.

Salas S \& Rigotti A. (2005). Médicos -Centíficos en Chile: ¿Una especie en extinción? Revista Médica de Chile, 133(1), 121-128.

Salas S \& Rigotti A. (2006). Los médicos científicos como especie universitaria en amenaza de extinción: Etiopatogenia, diagnóstico y tratamiento de una preocupante realidad académica. ARS MEDICA Revista de Ciencias Médicas, 35(2), 50-57.

Szot J. (2002). Reseña de la salud pública materno-infantil chilena durante los últimos 40 años: 1960-2000. Revista Chilena de Obstetricia Y Ginecología, 67(2), 129-135.

Varki A \& Rosenberg, L. (2002). Emerging opportunities and career paths for the young physician-scientist. Nature Medicine, 8(5), 437-439. 